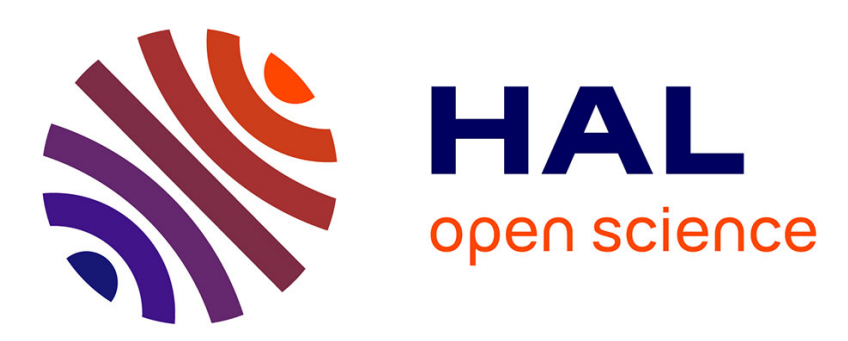

\title{
Transmission system using chaotic delays between lightwaves
}

Min Won Lee, L. Larger, Jp Goedgebuer

\section{To cite this version:}

Min Won Lee, L. Larger, Jp Goedgebuer. Transmission system using chaotic delays between lightwaves. IEEE Journal of Quantum Electronics, 2003, 39 (7), pp.931-935. 10.1109/JQE.2003.813223 . hal-00094864

\section{HAL Id: hal-00094864 \\ https://hal.science/hal-00094864}

Submitted on 26 Apr 2021

HAL is a multi-disciplinary open access archive for the deposit and dissemination of scientific research documents, whether they are published or not. The documents may come from teaching and research institutions in France or abroad, or from public or private research centers.
L'archive ouverte pluridisciplinaire HAL, est destinée au dépôt et à la diffusion de documents scientifiques de niveau recherche, publiés ou non, émanant des établissements d'enseignement et de recherche français ou étrangers, des laboratoires publics ou privés. 


\title{
Transmission System Using Chaotic Delays Between Lightwaves
}

\author{
Min Won Lee, Laurent Larger, and Jean-Pierre Goedgebuer
}

\begin{abstract}
We report a chaos-based transmission system that uses optical path differences between wavegroups produced by a short coherence source. Chaos synchronization is demonstrated in delay-differential equations involving optical delays larger than the coherence length of the source. The particular feature is all-optical chaos subtraction for message extraction and a very high masking efficiency.
\end{abstract}

Index Terms-Chaos, coherence modulation, encryption, unbalanced electrooptic Mach-Zehnder.

$\mathbf{I}$ N RECENT years, secure communication systems based on chaotic dynamics have known an increasingly interest in electronics [1], [2], optics [3], [4] and optoelectronics [5], [6]. Most investigations have focused on the problem of chaos synchronization [7] with low- and high-dimensional chaotic systems, and recently on the problem of anticipating chaos [8]. Also recently, all-optical chaos synchronization and signal encryption/decryption with signal bandwidths up to the gigahertz range have been demonstrated experimentally from chaotic lasers with external feedback [9], [10]. All of those investigations have been sustained by the need of exploring mechanisms by which coupled simple nonlinear elements can entrain one another, one of the last example in this area being the investigation of delay-coupled semiconductor lasers [11]. The basic idea behind the use of chaos to transmit messages is to mask a message within a chaotic carrier before transmission, which is a form of spreadspectrum communication, and makes the presence of the signal difficult to detect in either the time or frequency domain, although breaking methods have been recently proposed [12], [14]. The masking efficiency is typically $-15 \mathrm{~dB}$ in most of the present-day chaotic systems.

Unlike optical chaotic encryption systems reported to date, where chaos synchronization has been observed in the chaotic behavior of laser wavelength and laser intensity, we report in this paper the demonstration of chaos synchronization based on an unusual dynamical variable, the optical path-difference (OPD) between wavegroups of light produced by a short coherence source in coherence-modulated systems. Recent studies of chaos in coherence modulation have shown specific routes to chaos, including forward and reverse Hopf bifurcations, and their fundamental relevance [16]. We present the synchronization process

M. W. Lee is with the University of Wales, Bangor, Gwynedd LL57 2UW, Wales, U.K.

L. Larger and J.-P. Goedgebuer are with the University of Franche-Comté, 25030 Bensançon, France.

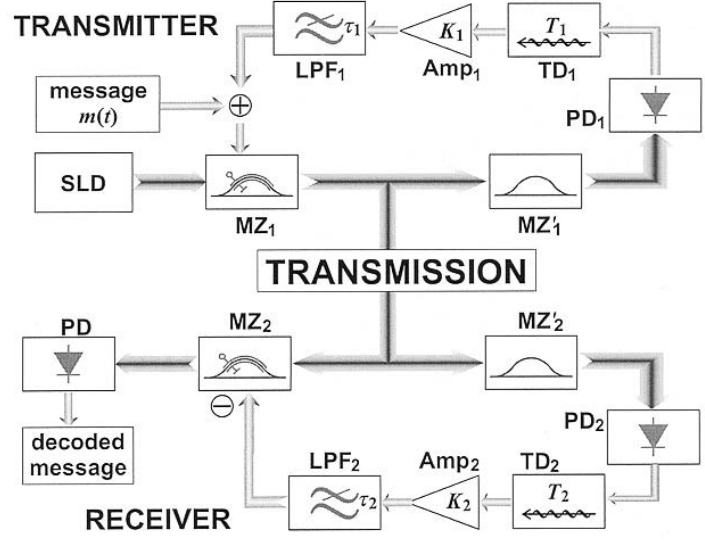

Fig. 1. Experimental setup. SLD: super luminescent diode. MZ: unbalanced electrooptic Mach-Zehnder. PD: photodiode. TD: time delay. Amp: amplifier. LPF: low-pass filter.

between two such chaos generators and report numerical and experimental results about its dependence on the chaotic keys.

The encryption system (Fig. 1) consists of a transmitter (subscript 1) and a receiver (subscript 2), which are built symmetrically. The message to be transmitted is $m(t)$. The transmitter consists of a short coherence source [a superluminescent diode (SLD)], and an integrated unbalanced Mach-Zehnder modulator $\left(\mathrm{MZ}_{1}\right)$ driven by a feedback loop. The feedback loop is formed by a second unbalanced $\mathrm{MZ}\left(\mathrm{MZ}_{1}^{\prime}\right)$, a photodetector $\mathrm{PD}_{1}$, a delay line (time delay $T_{1}=512 \mu \mathrm{s}$ ), an electronic amplifier (gain $K_{1}=20 \mathrm{~dB}$ ), and a low-pass filter $\mathrm{LPF}_{1}$ with a time response $\tau_{1}=6.4 \mu \mathrm{s}$ (which stands for the time constant of the setup and corresponds to a cut-off frequency $f_{c}$ ). The continuous wave (CW) SLD operates at $\lambda_{0}=\sigma_{0}^{-1} \approx 1.28 \mu \mathrm{m}$ and with a coherence length $L_{c} \approx 47 \mu \mathrm{m}$. Due to its low temporal-coherence degree, its emission may be regarded as a random sequence of wavegroups of coherence length $L_{c}$ and whose correlation function is related to the temporal coherence degree of the source [15, Ch. 10]. The interferometers $\mathrm{MZ}_{1}$ and $\mathrm{MZ}_{1}^{\prime}$ have identical static OPDs, $\left(D_{01}=D_{01}^{\prime}=260 \mu \mathrm{m}\right)$ greater than the coherence length. The optical beam at the output of $\mathrm{MZ}_{1}$ is therefore formed by random pairs of wavegroups that do not produce any detectable intensity modulation since their extension $L_{c}$ is much smaller than the OPD $D_{01}$ separating them (for more details about coherence modulation, we refer the reader to [16]). Electrooptically modulating $\mathrm{MZ}_{1}$ results in OPD variations $\Delta D_{1}(t)$ around $D_{01}$ that are linearly related to the modulation voltage $V_{1}(t)$ through $\Delta D_{1}=V_{1} /\left(2 V_{\pi 1} \sigma_{0}\right)$, where $V_{\pi 1}=4.3 \mathrm{~V}$ is the half-wave voltage of $\mathrm{MZ}_{1}$. The OPD at $\mathrm{Z}_{1}$ output is $D_{1}(t)=D_{01}+\Delta D_{1}(t)$, with $\Delta D_{1}(t) \ll D_{01}$. 
A part of this coherence-modulated beam is sent to the receiver, while the other part is launched in $\mathrm{MZ}_{1}^{\prime}$. $\mathrm{MZ}_{1}^{\prime}$ then converts nonlinearly the optical delay $D_{1}(t)$ into a detectable output intensity modulation given by [16]

$$
I_{1}(t)=P_{1}\left\{1+\frac{1}{2} \cos \left[2 \pi \sigma_{0}\left(D_{1}(t)-D_{10}^{\prime}\right)\right]\right\}
$$

where $P_{1} \approx 600 \mathrm{nW}$ is the average optical power at $\mathrm{MZ}_{1}^{\prime}$ output. After detection by $\mathrm{PD}_{1}$, the signal is time delayed by $T_{1}$ through the electronic delay line, filtered by the low-pass filter $\mathrm{LPF}_{1}$ and serves as the feedback signal applied to $\mathrm{MZ}_{1}$. The message $m(t)$ is added to the feedback signal [17] with its amplitude $(300 \mathrm{mV})$ at least ten times smaller than $V_{1}(t)(8.6 \mathrm{~V})$. The amplitude of the feedback voltage $V_{1}(t)$ is adjusted to be twice higher than $V_{\pi 1}$. Under these conditions, $\Delta D_{1}$ can be expressed as the addition of the OPD $\delta D_{1}(t)$ induced by the feedback signal and the OPD $D_{m}(t)$ induced by $m(t): \Delta D_{1}(t)=\delta D_{1}(t)+D_{m}(t)$ with $D_{m}=m(t) / 2 V_{\pi} \sigma_{0} \ll \delta D_{1}(t)$. Then, the transmitter equation, which rules the fluctuations of the OPD of $\mathrm{MZ}_{1}$ is a delayed differential equation (DDE) that can be shown to be [16]

$$
\delta D_{1}(t)+\tau_{1} \delta \dot{D}_{1}(t)=\mathrm{F}_{1}\left[\Delta D_{1}\left(t-T_{1}\right), \beta_{1}, \Phi_{1}\right] .
$$

In (2), $F_{1}$ is a nonlinear function derived from (1) and expressed as

$$
\mathrm{F}_{1}\left(D_{1}, \beta_{1}, \Phi_{1}\right)=\beta_{1}\left[1+\frac{1}{2} \cos \left(2 \pi \sigma_{0} D_{1}+\Phi_{1}\right)\right]
$$

where $\beta_{1}=2 K_{1} P_{1} / \sigma_{0} V_{\pi 1}=8$ is the bifurcation parameter and $\Phi_{1}=2 \pi \sigma_{0}\left(D_{01}-D_{01}^{\prime}\right)$. Finally, the message $m(t)$ is encrypted at the transmitter into a chaotic coherence-modulated beam formed by wavepackets separated by a chaotically modulated OPD $D_{1}(t)=D_{01}+\delta D_{1}(t)+D_{m}(t)$ larger than the coherence length $L_{c}$ of the source. Under these conditions, we do not have any detectable intensity modulation in the transmitter beam.

We have already described the complicated dynamics and routes to chaos corresponding to (2), showing that forward and reverse Hopf bifurcations can be obtained [16]. For the parameters used in the transmitter, the solutions of (2) are hyperchaotic, with the Lyapunov dimension evaluated from Kaplan-Yorke's conjecture [18], [19] found to be about 76.

The receiver is constructed from the same components as those in the transmitter. A part of the transmitted beam is sent into the loop of the receiver. This loop is formed by $\mathrm{MZ}_{2}^{\prime}$, which features a static OPD $D_{02}^{\prime}=D_{01}^{\prime}$ and a coherence demodulation function $\mathrm{F}_{2}$, a delay line $T_{2}$, an amplifier (gain $K_{2}$ ) and a low-pass filter $\mathrm{LPF}_{2}$ (response time $\tau_{2}$ ). The output electrical signal of the loop serves as the driving voltage of $\mathrm{MZ}_{2}$ (OPD $D_{02}$, half-wave voltage $V_{\pi 2}$ ) and generates chaotic fluctuations $\delta D_{2}$ of the OPD of $\mathrm{MZ}_{2}$ around $D_{02}$. Those chaotic fluctuations are ruled by a DDE that can be expressed as

$$
\delta D_{2}(t)+\tau_{2} \delta \dot{D}_{2}(t)=\mathrm{F}_{2}\left[\Delta D_{1}\left(t-T_{2}\right), \beta_{2}, \Phi_{2}\right]
$$

with $\beta_{2}=2 K_{2} P_{2} / \sigma_{0} V_{\pi 2}, \Phi_{2}=2 \pi \sigma_{0}\left(D_{01}-D_{02}^{\prime}\right), \mathrm{F}_{2}=$ $\beta_{2}\left[1+1 / 2 \cos \left(2 \pi \sigma_{0} D_{2}+\Phi_{2}\right)\right]$, and $P_{2}$ is the average optical power at $\mathrm{MZ}_{2}^{\prime}$ output. Assuming $\mathrm{F}_{2}=\mathrm{F}_{1}, \beta_{2}=\beta_{1}, \Phi_{2}=\Phi_{1}$, $\tau_{2}=\tau_{1}$, and $T_{2}=T_{1}$, (4) leads to solutions $\delta D_{2}(t)$ identical to

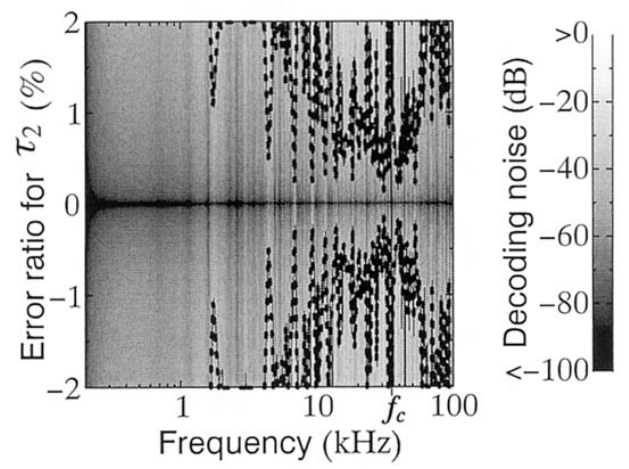

(a)

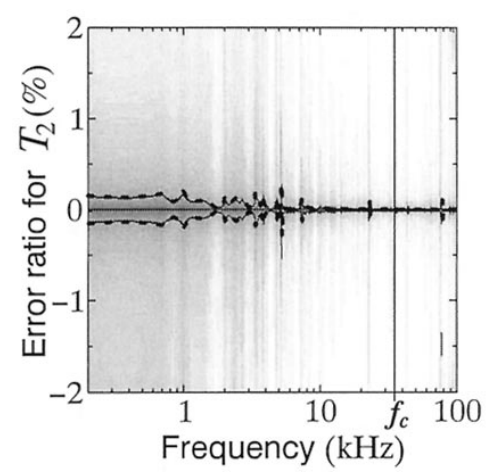

(b)

Fig. 2. Spectrum of the synchronization error for various parameter mismatches. The contour line indicates a $-40 \mathrm{~dB}$ noise level. (a) Response time $\tau$ mismatch. (b) Delay $T$ mismatch.

the solutions $\delta D_{1}(t)$ of (2), when there is no message ( $m=$ $0, D_{m}=0$ ) at the transmitter, meaning that we have chaos replication in that case.

The other part of the transmitted coherence-modulated beam is launched in $\mathrm{MZ}_{2} \cdot \mathrm{MZ}_{2}$ operates as a coherence demodulator that features a chaotically modulated OPD $D_{02}+\delta D_{2}(t)$ with $D_{02}=D_{01}+\lambda_{0} / 4$. That branch is aimed at optically carrying out a subtraction between the chaotic fluctuations $\Delta D_{1}(t)=$ $\delta D_{1}(t)+D_{m}(t)$ in the transmitted beam and those $\delta D_{2}(t)$ produced by $\mathrm{MZ}_{2}$, to retrieve the original message $m(t)$. Noting that $\delta D_{1}(t)=\delta D_{2}(t)$ and $D_{m}(t)$ is small, the output intensity $I_{2}(t)$ of $\mathrm{MZ}_{2}$ is given by

$$
I_{2}(t)=P_{2}\left\{1+\frac{1}{2} \sin \left[2 \pi \sigma_{0} D_{m}(t)\right]\right\} \approx P_{2}\left[1+\frac{\pi}{2 V_{\pi}} m(t)\right] .
$$

The original message $m(t)$ is then directly retrieved at the receiver output. Since matching the transmitter and receiver parameters is one key condition, it is important to know its impact on the recovery of the message. For that, we first evaluated the sensitivity of the synchronization process from numerical simulations using (2) and (4), and introducing voluntarily mismatches between the different parameters at the receiver. The computations were done with no message $\left(D_{m}(t)=0\right)$ and evaluating the power spectrum of the synchronization error signal $\delta D_{1}-\delta D_{2}$ (which is also chaotic), normalized to the rms level of the chaotic carrier $\left(\left\langle\delta D_{1}^{2}\right\rangle_{t}\right)$, as a function of the relative parameter mismatch expressed as a percent. Results of the simulations are plotted in Fig. 2 for mismatches ranging 


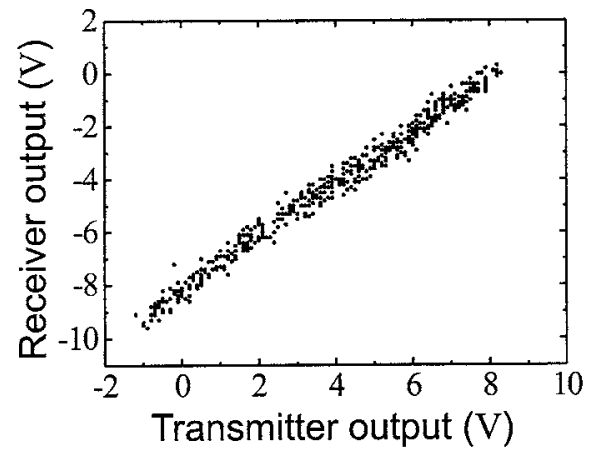

(a)

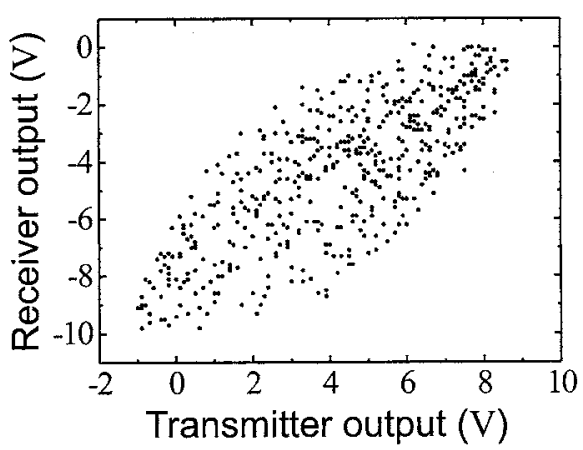

(b)

Fig. 3. Experimental synchronization with a mismatch between $T_{1}$ and $T_{2}$. (a) Receiver chaos versus emitter chaos for a $0.01 \%$-mismatch. (b) Same representation for a $1 \% T$-mismatch.

from $-2 \%$ to $2 \%$ for $\tau_{2}$ and for $T_{2}$. In Fig. 2, the magnitude of the decoding noise (expressed in decibels) is encoded into gray scales, where dark gray corresponds to a very small synchronization error (a good message recovery), whereas white indicates a very bad synchronization, and hence a bad message decoding. Typically, following the usual standards in telecommunications, a good decoding quality is obtained when the noise level is smaller than $-40 \mathrm{~dB}$ that corresponds here to $20 \mathrm{~dB}$ for the signal-to-noise ratio and $20 \mathrm{~dB}$ for the chaos-to-signal ratio. A contour for those $-40 \mathrm{~dB}$ is indicated in the figure. Computation shows that synchronization is very sensitive to a time delay mismatch [Fig. 2 (b)]. In that case, the decoding noise can reach $0 \mathrm{~dB}$ when the mismatch is a few percent, meaning that the message is completely altered by noise. In contrast, the synchronization process is much less sensitive to a $\tau$-mismatch, as shown in Fig. 2 (a). Similar computations can be done for parameters $\beta$ and $\Phi$, showing that an intermediate sensitivity is obtained, compared to the one presented for parameters $\tau$ and $T$.

To confirm those numerical simulations, we then investigated experimentally the synchronization process by varying the value of the time delay mismatch, all the other parameters being closely matched one with each other. The results are shown in Fig. 3. Fig. 3(a) shows, for a $0.01 \% T_{2}$-mismatch, the Lissajou representation of the driving signals of $\mathrm{MZ}_{1}$ and $\mathrm{MZ}_{2}$, which are linearly related to $\Delta D_{1}(t)$ and $\Delta D_{2}(t)$ at the transmitter and receiver, respectively. In spite of their multiple positive Lyapunov exponents [7], these signals show a very good synchronization, and the signal-to-noise ratio was then measured to be $36.5 \mathrm{~dB}$ from this figure. Fig. 3(b) corresponds to only a $1 \% T_{2}$-mismatch, resulting in a large synchronization error and non correlated chaotic signals. The fact that the most critical parameter to consider is the time delay in the feedback loop has already been observed with other chaotic schemes [20].

In a third step, we adjusted the parameters to meet the conditions of optimal matching (all the parameters matched to less than $1 \%$ ), and we introduced a message $m(t)$ in the feedback loop of the transmitter. The encryption and decryption results are illustrated in Fig. 4. Fig. 4(a) shows the original and decoded message $m(t)$ in the case of a $1-\mathrm{kHz}$ sine waveform. The driving voltage of $\mathrm{MZ}_{1}$ thus obtained (lower trace), and the intensity of the coherence-modulated beam carrying the message in the transmission line (upper trace), are shown in Fig. 4(b). The residual intensity modulation was measured to be smaller than $1 \%$ of the average intensity. This residual intensity modulation is due to the low mutual coherence degree of the wavegroups of light at $\mathrm{MZ}_{1}$ output, and could be made even smaller by using an OPD $D_{01}$ in $\mathrm{MZ}_{1}$ greater than $260 \mu \mathrm{m}$. We also show the spectrum of the residual intensity modulation of the coherence-modulated beam [Fig.4 (c)], which provides information that an eavesdropper can detect when tapping the transmission fiber. No detectable peak at the $1-\mathrm{kHz}$ message frequency can be seen in the spectrum, down to $-70 \mathrm{~dB}$, which is the masking efficiency (the masking efficiency in chaotic transmission systems is expressed as the message-to-chaos ratio in the transmitted signal). The message is therefore strongly hidden due to the chaotic coherence modulation encryption. Finally, the spectrum of the decoded message from the receiver is shown in Fig.4 (d), in which the signal-to-noise ratio was measured to be $33 \mathrm{~dB}$. In the case of a realistic broadband information message, we observed similar signal-to-noise ratio performances, which can be explained by the fact that the decoding noise does not depend significantly on the frequency inside the chaos generator bandwidth (see Fig. 2).

To summarize, we have reported for the first time the proofs of principle of an optical cryptographic system based on a chaotic coherence modulation. OPD fluctuations are used to mask an information message. The chaotic waveform exhibits a high Lyapunov dimension $(\approx 76)$. Such a hyperchaos was successfully synchronized in a transmitter and receiver, and the impact of mismatches has been investigated numerically and experimentally. Encryption and decryption of a message was obtained experimentally with a 33-dB signal-to-noise ratio. Direct recovery of the message at the receiver was performed through a subtraction process performed optically (which seems to be specific to the coherence demodulation scheme), unlike other schemes in which an electronic subtractor is generally used. The system features an extremely high masking efficiency $(-70 \mathrm{~dB})$, a value which is the best performance reported with chaotic systems so far. The system could operate in the gigahertz range with high-speed components [9]. Another attractive feature of the system concerns the multiplexing capabilities of coherence modulation systems [21], in which each channel can be attached to a different OPD. Finally, the system can be potentially dedicated to optical telecommunications, 


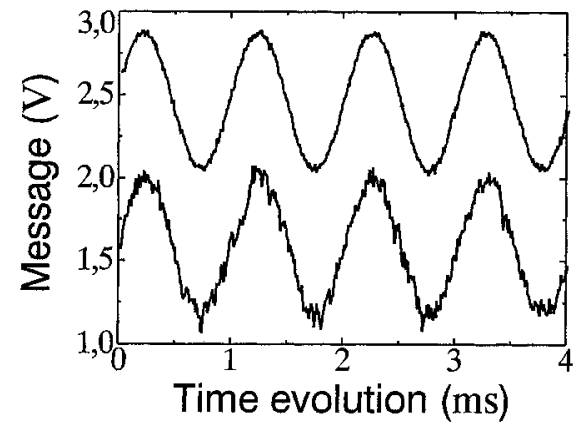

(a)

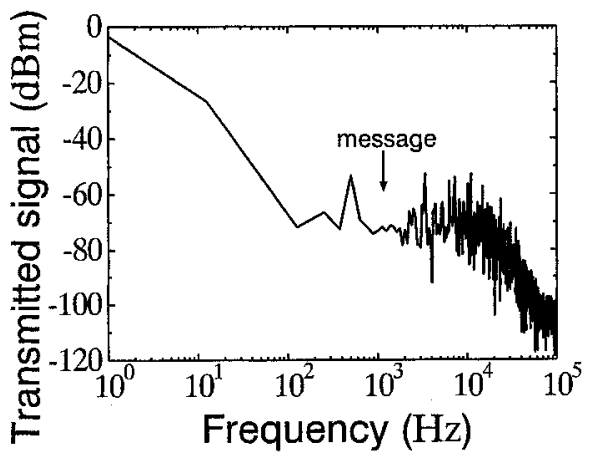

(c)

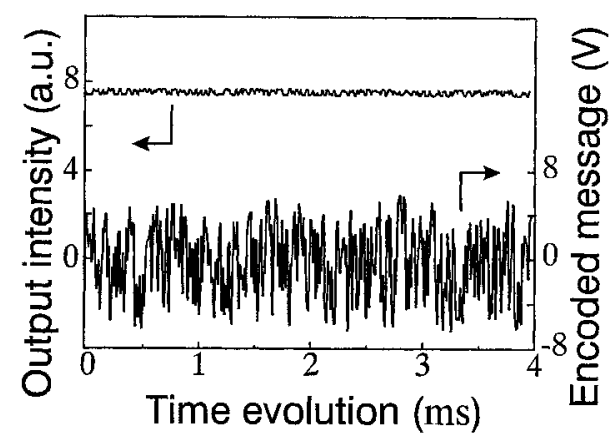

(b)

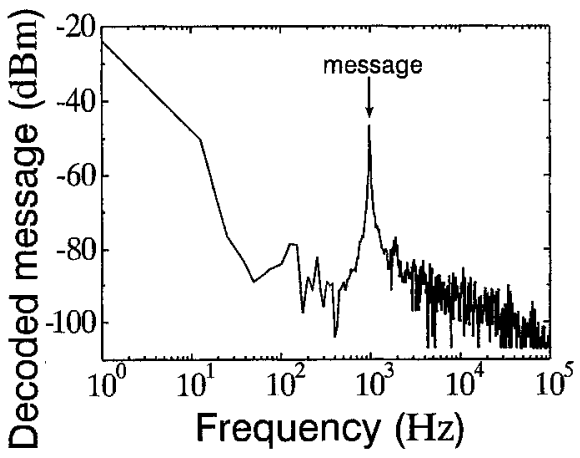

(d)

Fig. 4. Experimental encoding and decoding results. (a) Original and decoded 1-kHz sine-waveform. (b) Transmitted intensity and corresponding OPD fluctuations. (c) FFT of the transmitted intensity. (d) FFT of the recovered message.

since it makes use of fiber-based components and integrated Mach-Zehnder modulators.

\section{REFERENCES}

[1] L. M. Pecora and T. L. Carroll, "Synchronization in chaotic systems," Phys. Rev. Lett., vol. 64, pp. 821-824, 1990.

[2] K. M. Cuomo and A. V. Oppenheim, "Circuit implementation of synchronized chaos with applications to communications," Phys. Rev. Lett., vol. 71, pp. 65-68, 1993.

[3] G. D. VanWiggeren and R. Roy, "Optical communication with chaotic waveforms," Phys. Rev. Lett., vol. 81, pp. 3547-3550, 1998.

[4] H. Fujino and J. Ohtsubo, "Experimental synchronization of chaotic oscillations in external-cavity semiconductor lasers," Opt. Lett., vol. 25, pp. 625-627, 2000.

[5] L. Larger, J.-P. Goedgebuer, and F. Delorme, "Optical encryption system using hyperchaos generated by an optoelectronic wavelength oscillator," Phys. Rev. E, vol. 57, pp. 6618-6624, 1998.

[6] A. Uchida, S. Yoshimori, T. Shinozuka, and F. Kannari, "Chaotic on off keying for secure communication," Opt. Lett., vol. 26, pp. 866-868, 2001.

[7] J. H. Peng, E. J. Ding, M. Ding, and W. Yang, "Synchronizing hyperchaos with a scalar transmitted signal," Phys. Rev. Lett., vol. 76, pp. 904-907, 1996.

[8] H. U. Voss, "Anticipating chaotic synchronization," Phys. Rev. E, vol. 61, pp. 5115-5119, 2000.

[9] I. Fischer, Y. Liu, and P. Davis, "Synchronization of chaotic semiconductor laser dynamics on subnanosecond time scales and its potential for chaos communication," Phys. Rev. A, vol. 62, no. 011801, 2000.

[10] S. Tang and J. M. Liu, "Message encoding and decoding at $2.5 \mathrm{Gbits} / \mathrm{s}$ through synchronization of chaotic pulsing semiconductor laser," Opt. Lett., vol. 26, pp. 1843-1845, 2001.

[11] S. Sivaprakasam and K. A. Shore, "Message encoding and decoding using chaotic external-cavity diode lasers," IEEE J. Quantum Electron., vol. 36, pp. 35-39, 2000.

[12] J. B. Geddes, K. M. Short, and K. Black, "Extraction of signals from chaotic laser data," Phys. Rev. Lett, vol. 83, pp. 5389-5392, 1999.

[13] C. Zhou and C.-H. Lai, "Extracting messages masked by chaotic signals of time-delay systems," Phys. Rev. E, vol. 60, pp. 320-323, 1999.
[14] R. Hegger, M. J. Bünner, and H. Kantz, "Identifying and modeling delay feedback systems," Phys. Rev. Lett., vol. 81, pp. 558-561, 1998.

[15] M. Born and E. Wolf, Principles of Optics, 7th ed. Cambridge, U.K.: Cambridge Univ. Press, 1999, p. 564.

[16] L. Larger, M. W. Lee, J.-P. Goedgebuer, T. Erneux, and W. Elflein, "Chaos in coherence modulation: bifurcations of an oscillator generating optical delay fluctuations," J. Opt. Soc. Amer. B, vol. 18, pp. 1063-1068, 2001.

[17] V. S. Udaltsov, J.-P. Goedgebuer, L. Larger, and W. T. Rhodes, "Communicating with optical hyperchaos: information encryption and decryption in delayed nonlinear feedback systems," Phys. Rev. Lett., vol. 86, pp. 1892-1895, 2001.

[18] J. D. Farmer, "Chaotic attractors of an infinite-dimensional dynamical system," Physica 4D, pp. 366-393, 1982.

[19] J. Kaplan and J. Yorke, Functional Differential Equations and Approximation of Fixed Points. Berlin, Germany: Springer, 1978, p. 228.

[20] J.-B. Cuenot, L. Larger, J.-P. Goedgebuer, and W. T. Rhodes, "Chaos shift keying with an optoelectronic encryption system using chaos in wavelength," J. Quantum Electron., vol. 37, pp. 849-855, 2001.

[21] J. Hauden, H. Porte, and J.-P. Goedgebuer, "Demonstration of a single source bidirectional fiber link using polarization insensitive $\mathrm{LiNbO}_{3}$ integrated coherence modulator," Electron. Lett., vol. 32, pp. 751-752, 1996. 